\title{
Octave-spanning Coherent Mid-IR Pulses via Adiabatic Difference Frequency Generation
}

\author{
Jeffrey Moses ${ }^{1}$, Peter R. Krogen ${ }^{1}$, Shu-Wei Huang ${ }^{1}$, Franz X. Kärtner ${ }^{1,2}$ and Haim Suchowski ${ }^{3}$ \\ ${ }^{I}$ Department of Electrical Engineering and Computer Science and Research Laboratory of Electronics, Massachusetts Institute of Technology, \\ Cambridge, Massachusetts 02139, USA \\ ${ }^{2}$ Center for Free-Electron Laser Science, DESY and Physics Department University of Hamburg, Notkestraße 85, D-22607 Hamburg, Germany \\ ${ }^{3}$ NSF Nanoscale Science and Engineering Center, University of California, Berkeley, California 94720, USA \\ j_moses@mit.edu
}

\begin{abstract}
We generate a $2.05-4.6-\mu \mathrm{m}$ mid-IR spectrum $(-10 \mathrm{~dB})$ by adiabatic conversion of a near-IR OPA pulse, employing a single 2-centimeter quasi-phase-matched lithium niobate grating. The technique is widely applicable for ultrabroadband near-IR to mid-IR conversion.

OCIS codes: (320.7110) Ultrafast Nonlinear Optics; (320.6629) Supercontinuum Generation
\end{abstract}

The fields of nonlinear infrared spectroscopy [1] and strong-field laser-matter interaction science [2] demand broadband, coherent, energetic sources of mid-IR light. Broadband amplifiers covering the 2-5 $\mu \mathrm{m}$ range are currently lacking. Success has been achieved to some degree by several candidate technologies, including optical parametric amplifiers and doubly resonant optical parametric oscillators, conventional difference frequency generation, and plasma generation driven by mid-IR or UV/VIS light pulses [3]. As of yet, each of these technologies for producing broadband mid-IR light is currently insufficient in some respect for producing multioctave spanning, energetic ( $\mu \mathrm{J}$ to $\mathrm{mJ})$ sources with relatively flat spectral power distribution.

Here we present the proof of principle of a new approach for the efficient conversion of broadband near-IR sources to ultrabroad mid-IR bands. We demonstrate downconversion of a near-IR broadband optical parametric chirped pulse amplifier (OPCPA) pulse to the mid-IR via an adiabatic frequency converter [4] based on a single nonlinearly chirped quasi-phase-matching grating in lithium niobate. We report a spectrum spanning an octave from 2.05 to $4.6 \mu \mathrm{m}$ at $-10 \mathrm{~dB}$ of peak, and obtained by near full photon number conversion of a 5- $\mu \mathrm{J}$ OPCPA pulse spanning $680-870 \mathrm{~nm}$ mixed with a narrowband $1047-\mathrm{nm}$ pulse. This is achieved by a highly practical adiabatic difference frequency generation (ADFG) device that may be applied as a post-amplification converter for many near-IR systems. Advantages of the technique are many-fold. It can produce a bandwidth far overcoming the typical phase-matching limitations of nonlinear optical frequency converters; it allows full photon-number conversion efficiency for inhomogeneous beam and pulse profiles, allowing a much higher conversion efficiency than standard techniques; it preserves signal amplitude modulation during conversion, and transfer of signal phase to the mid-IR, thus offering fidelity and convenience for applications requiring phase coherence; it has the ability to convert well established high-energy and broadband near-IR sources to the mid-IR, making it highly relevant to established laser systems; and it possesses a simple design consisting of only a single crystal and a few optics, allowing the potential for compactness of system design.

Our experimental setup is illustrated in Fig. 1. A Ti:sapphire oscillator injection seeded a home-built, 12-ps, 1$\mathrm{kHz}, 4-\mathrm{mJ} \mathrm{Nd}$ :YLF chirped pulse amplifier (CPA) [5] used as an OPCPA pump after second harmonic generation (SHG) in LBO. The resulting 1-mJ, 8-ps, 523-nm pulses pumped a noncollinear OPCPA that we designed for this demonstration (single-stage, 5 -mm BBO, $\theta=24.0^{\circ}$ and $\alpha=2.4^{\circ}$ ) seeded by the same Ti:sapphire oscillator. The seed pulses for the OPCPA were first stretched by a double pass through a grism pair. A single-pass gain of $\sim 10^{5}$ resulted in $5-\mu \mathrm{J}$ energy at $1-\mathrm{kHz}$ repetition rate. A $48-\mathrm{mm}$ SF10 glass block was then used to flip the sign of the pulse chirp from negative to positive to avoid self-compression in the ADFG stage. The resulting $\sim 3$-ps near-IR pulses (the ADFG "signal") were then combined collinearly at a dichroic mirror with $\sim 0.6 \mathrm{~mJ}$ of the remaining Nd:YLF pulse energy at $1047 \mathrm{~nm}$ (the ADFG "pump") and sent to an aperiodically poled, MgO-doped congruent lithium niobate (APMCLN) grating for broadband ADFG.

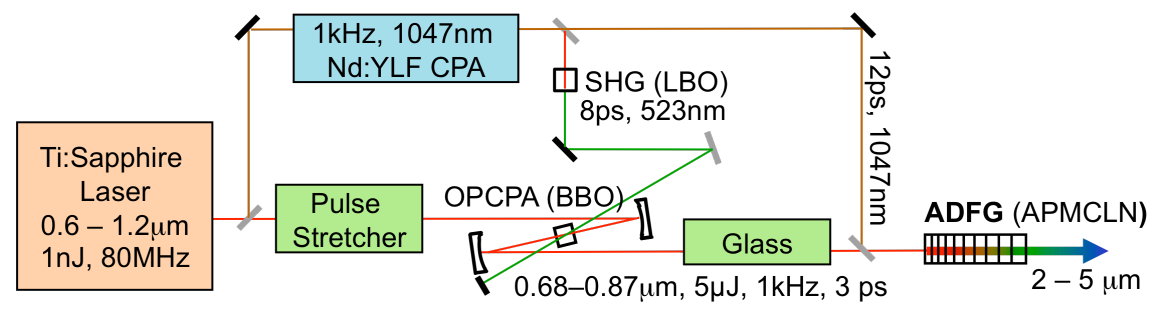

Fig. 1. Experimental setup for adiabatic difference frequency conversion of OPCPA pulses. 
ADFG requires a suitably small longitudinal variation in wave-vector mismatch and a pump intensity much greater than the signal intensity to achieve the conditions for complete adiabatic passage of photon number from a signal to an idler (i.e., the difference of signal and pump frequencies) [4]. For each signal frequency, the phase mismatch is swept through zero at a different distance in the crystal. This technique was employed previously for conversion of nanojoule Ti:sapphire oscillator pulses to an ultrabroadband near-IR spectrum [6], and for the generation of an 0.2-octave pulse at $3.15 \mu \mathrm{m}$ [7]. In the present work, our novel system and device design allows the first demonstration of energetic and octave-spanning mid-IR generation. We employ a nonlinear variation of poling period from 12-24 $\mu \mathrm{m}$ over a 2 -cm grating length to satisfy conditions for complete adiabatic conversion of a 0.66 $0.87-\mu \mathrm{m}$ signal band to a $1.8-5.0-\mu \mathrm{m}$ idler band for a pump intensity threshold of $\sim 5 \mathrm{GW} / \mathrm{cm}^{2}$. It is the beauty of the adiabatic approach to allow in a single grating the simultaneous conversion of all frequencies that can be quasiphase-matched in a given medium (and for a given choice of signal, pump, and idler polarizations), as long as a grating with wide enough poling period variation can be manufactured.

With a pump intensity of $27 \mathrm{GW} / \mathrm{cm}^{2}$, we observed uniform spectral power depletion of the near-IR spectrum, indicating nearly complete photon number conversion from signal to idler and corresponding to a signal to idler energy conversion ratio of $\sim 1 / 4$. The mid-IR power was collected by a scanning monochromator with a TE-cooled $\mathrm{PbSe}$ photodetector (Horiba/JY). The relative spectral intensity response of the monochromator and photodetector were calibrated with a black-body source, and a Ge filter was employed to block residual pump light that could appear from multiple-order grating reflections. Fig. 2 shows the normalized measured power spectrum (solid red curve) alongside the normalized "expected" power spectral density (dashed black curve) calculated by assuming $100 \%$ conversion of the OPCPA power spectrum (top right inset) to the mid-IR via ADFG and the varying quantum defect, $\lambda_{\text {signal }} / \lambda_{\text {idler }}$. We observe a direct transfer of the near-IR spectral amplitude profile to the mid-IR, spanning more than an octave $(2.05-4.6 \mu \mathrm{m})$ at $-10 \mathrm{~dB}$ of peak. As a practical aside, we note that by altering the OPCPA spectrum, the resulting mid-IR spectral profile can be tailored as needed for specific applications.

In conclusion, we have demonstrated an octave-spanning mid-IR source of approximately $1 \mu \mathrm{J}$ energy by adiabatic frequency conversion of near-IR OPCPA pulses, in a scheme we expect will have broad applicability as a post-amplification method for near-IR to mid-IR conversion and potentially allowing single-cycle pulsed sources. Our next step is confirmation of the expected direct transfer of spectral phase profile from near-IR to mid-IR, and pulse compression.

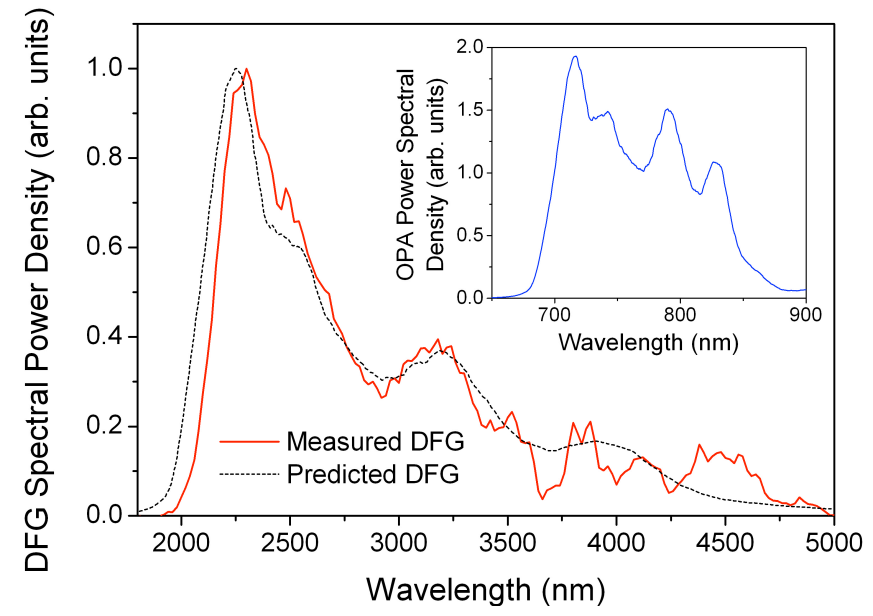

Fig. 2. The measured and predicted octave-spanning mid-IR ADFG spectrum. Near-IR spectrum (inset) also shown.

[1] P. Hamm and M. T. Zanni, Concepts and methods of 2d infrared spectroscopy (Cambridge University Press, 2011).

[2] P. Colosimo, G. Doumy, C. I. Blaga, J. Wheeler, C. Hauri, F. Catoire, J. Tate, R. Chirla, A. M. March, G. G. Paulus, H. G. Muller, P. Agostini, and L. F. DiMauro, "Scaling strong-field interactions towards the classical limit," Nat. Physics 4, 386-389 (2008).

[3] A. Schliesser, N. Picqué, and T. W. Hänsch, "Mid-infrared frequency combs," Nat. Photon. 6, 440-449 (2012).

[4] H. Suchowski, D. Oron, A. Arie, and Y. Silberberg, "Geometrical representation of sum frequency generation and adiabatic frequency conversion," Phys. Rev. A 78, 063821 (2008).

[5] J. Moses, S.-W. Huang, K.-H. Hong, O. D. Mücke, E. L. Falcao-Filho, A. Benedick, F. Ö. Ilday, A. Dergachev, J. A. Bolger, B. J. Eggleton, and F. X. Kärtner, "Highly stable ultrabroadband mid-IR optical parametric chirped-pulse amplifier optimized for superfluorescence suppression," Opt. Lett. 34, 1639-1641 (2009).

[6] J. Moses, H. Suchowski, and F. X. Kärtner, "Fully efficient adiabatic frequency conversion of broadband Ti:sapphire oscillator pulses," Opt. Lett. 37, 1589-1591 (2012).

[7] H. Suchowski, B. D. Bruner, A. Ganany-Padowicz, I. Juwiler, A. Arie, and Y. Silberberg, "Adiabatic frequency conversion of ultrafast pulses,” Appl. Phys. B 105, 697-702 (2011). 